\title{
Synergistic effects of baicalein with gemcitabine or docetaxel on the proliferation, migration and apoptosis of pancreatic cancer cells
}

\author{
PAN LIU ${ }^{1 *}$, JIARUI FENG $^{2 *}$, MENG SUN $^{1 *}$, WEN YUAN $^{3}$, RUIJING XIAO $^{3}$, JIE XIONG $^{3}$, XIAOXIN HUANG ${ }^{4}$, \\ MENG XIONG ${ }^{3}$, WU CHEN ${ }^{5}$, XIN YU $^{5}$, QIAN SUN ${ }^{5}$, XIAOJIE ZHAO ${ }^{5}$, QIUPING ZHANG ${ }^{3}$ and LIANG SHAO ${ }^{1}$ \\ ${ }^{1}$ Department of Hematology, Zhongnan Hospital of Wuhan University, Wuhan, Hubei 430071; ${ }^{2}$ Department of Hepatobiliary \\ and Laparoscopic Surgery, Renmin Hospital of Wuhan University, Wuhan, Hubei 430060; ${ }^{3}$ Department of Immunology,

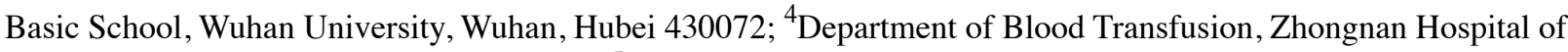 \\ Wuhan University, Wuhan, Hubei 430071; ${ }^{5}$ Basic School, Wuhan University, Wuhan, Hubei 430072, P.R. China
}

Received April 2, 2017; Accepted September 18, 2017

DOI: $10.3892 /$ ijo.2017.4153

\begin{abstract}
Baicalein, a type of flavonoids extracted from Scutellaria baicalensis Georgi, has been reported to be a very promising drug for pancreatic cancer. However, it is unclear whether combination of baicalein with gemcitabine or docetaxel is synergistic to the treatment for pancreatic cancer (PC). We investigated the combinational effects of baicalein with gemcitabine or docetaxel on proliferation, cell cycle, migration and apoptosis of human PC cells. Administration of baicalein alone significantly inhibit the proliferation of PC cells. Notably, when it is combined with gemcitabine or docetaxel, combination index (CI) values calculated by Calcusyn software are smaller than 1 , indicating the synergism of baicalein with gemcitabine or docetaxel for the treatment of PC cells. Consistently, EdU assay showed that administration of baicalein significantly enhanced the capacity of gemicitabine to inhibit proliferation of PC cells. Cell cycle analysis showed that high-concentration of baicalein was able to arrest $\mathrm{PC}$ cells in the $\mathrm{S}$ phase. Furthermore, low concentration of baicalein in combination with either gemcitabine or docetaxel exhibited strong suppression on the migration of PC cells. A further study using transmission electron microscope (TEM), DAPI staining and western blot showed that baicalein inducedapoptosis of PC cells might be via caspase-3/PARP signaling pathway. Notably, combination treatment was able to induce
\end{abstract}

Correspondence to: Dr Liang Shao, Department of Hematology, Zhongnan Hospital of Wuhan University, 169 Donghu Road, Wuchang, Wuhan, Hubei 430071, P.R. China

E-mail: liangsmd@163.com

*Contributed equally

Key words: pancreatic cancer, proliferation, migration, apoptosis, baicalein, gemcitabine, docetaxel more severe cell apoptosis of PC cells. In conclusion, baicalein exhibited synergistic effects with gemcitabine or docetaxel on the treatment of PC cells.

\section{Introduction}

Pancreatic cancer (PC) is a devastating disease with a 5-year survival rate $<7 \%(1,2)$. In most cases, the initial diagnosis of PC is made when the disease has progressed to a late stage, thus a poor prognosis is inevitable. Although surgery has made great progress to improve the overall survival of PC patients, the majority still survive less than 5 years (2-5). According to National Comprehensive Cancer Network (NCCN) criteria, combination therapy of gemcitabine with paclitaxel is currently the best choice for PC patients. Gemcitabine is a nucleoside analogue widely used as an important drug against carcinoma (6). Docetaxel, a semisynthetic analogue of paclitaxel, is a widely used anti-mitotic drug for various cancers, including PC (7). However, the combination regimen of gemcitabine plus paclitaxel is still unsatisfactory in the case of overall survival (OS), and severe side effects are common (8). Therefore, an alternative approach with minimal drug-related side effects to increase the OS of PC patients is urgently needed.

Baicalein, a kind of traditional Chinese herbal medicine, has been reported to be a potent chemotherapeutic adjuvant for its properties of selectively inducing apoptosis in various human cancer cells with minimal influence on normal cells (9-15). It has been demonstrated that baicalein has the capacity to induce apoptosis and inhibits the proliferation of PC cells in pre-clinical studies $(10,16,17)$. Furthermore, baicalein is able to activate caspase- 3 by forming hydrogen bonds with residues Ser251 and Asp253 at its active site and results in cell apoptosis in colon cancer (14). However, whether baicalein has synergistic effects with gemcitabine or docetaxel in the treatment of pancreatic cancer is still unclear. In the current study, we examined the effects of combination therapy of baicalein with gemcitabine or docetaxel on proliferation, apoptosis, migration, and cell cycle of pancreatic cells 
in vitro. Furthermore, we gained insight into the underlying mechanism of combination treatment containing baicalein. Our results suggest that synergistic effects of baicalein with gemcitabine or docetaxel on apoptosis of PANC-1 cells is dependent on caspase-3/PARP signaling pathway.

\section{Materials and methods}

Cell lines and reagents. Human pancreatic cell line PANC-1, MIA PaCa-2 and HPAF-II were purchased from Chinese Academy of Life Science (Shanghai, China). DMEM medium was purchased from HyClone Laboratories Inc. (Shrewsbury, NJ, USA). Fetal bovine serum (FBS) was from Gibco Co. (Carlsbad, CA, USA). Baicalein was purchased from Xinran Co. (Shanghai, China). Gemcitabine and docetaxel were purchased from Sigma-Aldrich (St. Louis, MO, USA) and were dissolved according to the manufacturer's instructions. DMSO and DAPI were from Roche.

Cell culture. PANC-1, MIA PaCa-2 and HPAF-II cells were cultured in DMEM medium containing 10\% FBS and $1 \%$ penicillin and streptomycin in incubator with $5 \% \mathrm{CO}_{2}$ at $37^{\circ} \mathrm{C}$. Drugs at different dosage were given at the indicated time point.

\section{3-(4, 5-Dimethylthiazol-2-yl)-2, 5-diphenyltetrazolium (MTT)} assay. Drug sensitivity was detected using the MTT assay. Briefly, cells were trypsinized and seeded in 96-well plates (Corning Inc., Corning, NY, USA) at $5 \times 10^{3}$ cells per well. The cells were cultured overnight and then replenished with fresh medium containing drugs at indicated concentrations. The cells were then incubated for $48 \mathrm{~h}$. A total of $20 \mu \mathrm{l}$ of MTT (Sigma-Aldrich) dissolved in PBS at $5 \mathrm{mg} / \mathrm{ml}$ was added directly to the wells at the indicated time points. The plates were then incubated for an additional $4 \mathrm{~h}$ at $37^{\circ} \mathrm{C}$ for MTT reaction. The supernatant was then removed. A total of $100 \mu \mathrm{l}$ of DMSO was added to dissolve the formed formazan crystals, and the optical density was measured at $490 \mathrm{~nm}$ on a PerkinElmer 2030 VICTOR X Multilabel Plate Reader (Perkin-Elmer, Waltham, MA, USA). The results are represented as the average value of 3 independent experiments. The percentage of viable cells was calculated as cell viability $(\%)=(\mathrm{OD}$ of treatment/OD of control $) \times 100$.

Analysis of cytotoxic synergy. The viability of PANC-1, MIA PaCa-2 and HPAF-II cells were examined by MTT assay as described above and the CI values were analyzed using the Calcusyn software, which calculates CI value by the following equation: $\mathrm{CI}=(\mathrm{D}) 1 /(\mathrm{Dx}) 1+(\mathrm{D}) 2 /(\mathrm{Dx}) 2+(\mathrm{D}) 1(\mathrm{D}) 2 /(\mathrm{Dx}) 1(\mathrm{Dx}) 2$, where (Dx)1 and (Dx)2 are the doses for $\mathrm{x} \%$ inhibition by drug 1 and drug 2 alone. (D)1 and (D) 2 are the doses in combination that inhibit cell growth by $\mathrm{x} \%$. A CI value of 1 indicates additive effects of the two agents, while a CI value greater than 1 indicates antagonism effects, and less than 1 indicates synergism effects.

DAPI staining assay. Viable PANC-1 cells were plated in 6-well plates for $24 \mathrm{~h}$, followed by treatment with indicated drugs. After $48 \mathrm{~h}$, cells were fixed with $4 \%$ paraformaldehyde for $20 \mathrm{~min}$, followed by DAPI staining for $10 \mathrm{~min}$ in the dark. Finally, cells were detected using immunofluorescence microscopy (DSY5000X, OPPNO, Beijing, China).

EdU (5-ethynyl-2'-deoxyuridine) assay. Cell Light ${ }^{\mathrm{TM}}$ EdU kit was purchased from RiboBio Co., Ltd. (Guangzhou, China) and the experiment was conducted according to the manufacturer's instructions. Briefly, prepared $50 \mu \mathrm{M}$ EdU DMEM medium solutions were added to drug treated PANC-1 cells in 96-well plates, followed by incubation for $2 \mathrm{~h}$ and thereafter washing with PBS, 4\% paraformaldehyde was used to fix the cells for $30 \mathrm{~min}$, and then $2 \mathrm{mg} / \mathrm{ml}$ glycine was given to neutralize the remaining paraformaldehyde. Apollo ${ }^{\circledR}$ staining reaction solution was used to incubate PANC-1 cells in the dark for $30 \mathrm{~min}$, and then washed with $0.5 \%$ TritonX-100 PBS 3 times. Finally, Hoechst 33342 was added for $30 \mathrm{~min}$ and images were taken from immunofluorescence microscopy (DSY5000X, OPPNO).

Wound healing assay. PANC-1 cells were seeded on 6-well plates (Corning Inc.). After incubation for $24 \mathrm{~h}$, each well was initiated by scratching with a sterile $10 \mu \mathrm{l}$ pipette tip, followed by washing with PBS three times, and treated with indicated drugs at $37^{\circ} \mathrm{C}$. After $24 \mathrm{~h}$, the distance between cell edges was analyzed using the ImageJ software.

Flow cytometry. After treatment with drugs, PANC-1 cells were digested by $0.25 \%$ trypsin from 6 -well plates, and then collected and incubated with $70 \%$ ethanol overnight. After fixation, cells were stained with propidium iodide (PI) for $30 \mathrm{~min}$ at room temperature. The cells were washed with ice cold PBS 3 times before loaded to the flow cytometer (FACS Calibur, BD BioSciences). The results were then analyzed with ModFit software according to the manufacturer's instructions.

Transmission electron microscope (TEM). PANC-1 cell samples were digested by $0.25 \%$ trypsin and centrifuged at $1500 \mathrm{rpm}$ for $5 \mathrm{~min}$ and fixed overnight in $2.5 \%$ glutaraldehyde at $4^{\circ} \mathrm{C}$. Then the samples were fixed in $1 \%$ osmium acid, dehydrated, and placed in embedding molds in a standard fashion. Appropriate areas were selected and ultrathin sections of $0.08 \mu \mathrm{m}$ were stained with lead citrate and uranyl acetate. Those sections were then examined with a transmission electron microscope (JEM1230, JEOL, Ltd., Tokyo, Japan).

Western blotting. PANC-1 cells were treated with drugs for $48 \mathrm{~h}$ and lysed in RIPA buffer, followed by denaturation. Protein concentration was measured by bicinchoninic acid assay system (Beyotime, Shanghai, China). Protein samples were separated by SDS-PAGE gel and electrophoretically transferred to nitrocellulose membranes. The membranes were blocked with $5 \%$ non-fat milk for 30 min and incubated with anti-caspase-3, anti-cleaved-caspase-3, anti-PARP and anticleaved-PARP antibodies (1:1,000; Cell Signaling Technology, Danvers, MA, USA) at $4^{\circ} \mathrm{C}$ overnight. The membranes were then incubated with goat anti-rabbit/anti-mouse secondary antibody conjugated with horseradish peroxidase (1:3,000; Cell Signaling Technology) and then membranes detected using an enhanced chemiluminescence detection kit (Thermo Scientific). $\beta$-actin was used as the internal control. 

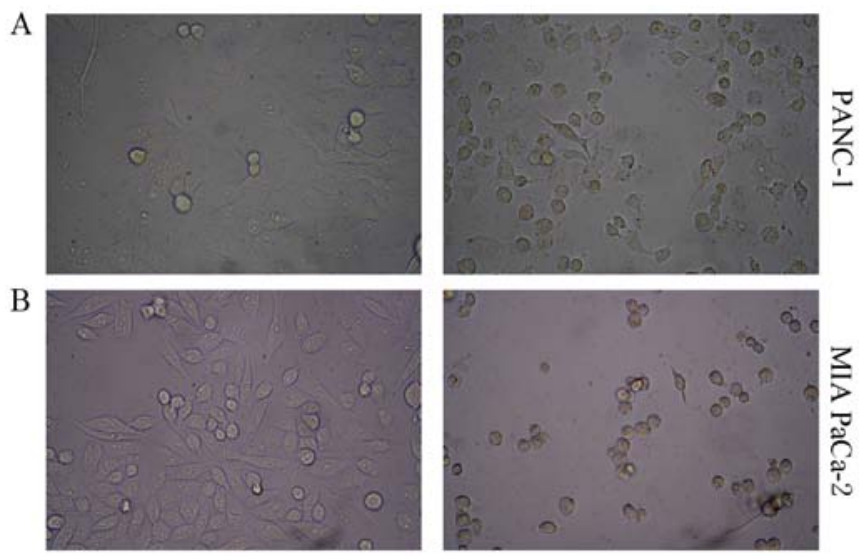

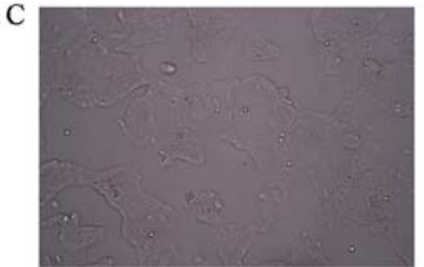

DMSO

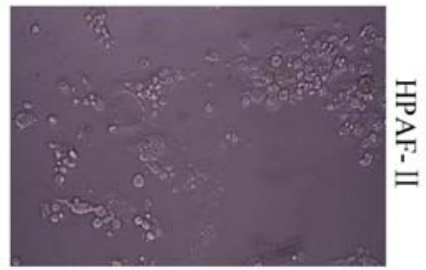

Baicalein $(200 \mu \mathrm{M})$
D

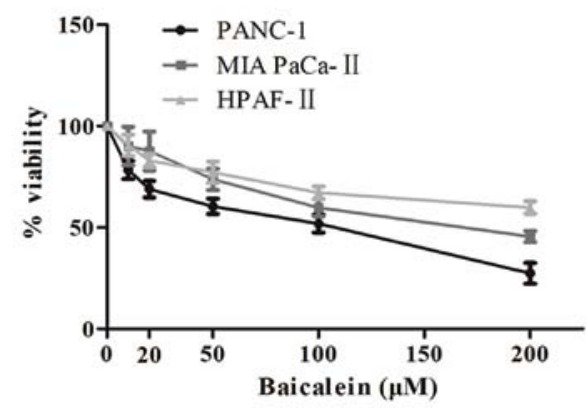

Figure 1. Effects of baicalein on the morphological change and cell viability of PANC-1, MIA PaCa-2 and HPAF-II cells. (A) Morphological change of baicalein-treated PANC-1 cells was visualized by inverted optical microscope. After treatment with drugs for $48 \mathrm{~h}$ at indicated concentration, morphology of PANC-1 cells was detected by inverted optical microscope. The images were taken at $\mathrm{x} 400$ magnification. (B) Morphological change of baicalein-treated MIA PaCa-2 cells was visualized by inverted optical microscope. The images were taken at $\times 400$ magnification. (C) Morphological change of baicalein-treated HPAF-II cells was visualized by inverted optical microscope. The images were taken at $x 400$ magnification. (D) Viabilities of PANC-1, MIA PaCa-2 and HPAF-II cells were determined by MTT assay after treatment with baicalein at indicated concentrations.

Statistical analysis. For statistical analyses, Prism 5 software was used. Statistical analyses were performed using the Student's t-test. A P-value of $<0.05$ was considered to indicate a statistically significant difference.

\section{Results}

Baicalein induced morphologic changes of pancreatic cancer cells and suppressed their proliferation. We determined the morphologic changes of PANC-1, MIA PaCa-2 and HPAF-II cells after treatment with baicalein $(200 \mu \mathrm{M})$ by inverted microscope. After treatment, cells exhibited a more distinct cell profile and more shrunken morphology, while DMSO-treated control group showed a blurred cell profile and adhered tightly to the well (Fig. 1A-C). Then, we detected the effect of baicalein on cell viability in vitro using MTT assay. Consistently, cell viability of baicalein-treated PANC-1 was significantly suppressed (Fig. 1D). As shown in Fig. 1D, $200 \mu \mathrm{M}$ baicalein triggered significant decrease in cell viability. Notably, the suppressive effects of baicalein on cell viability of PANC-1, MIA PaCa-2 and HPAF-II cells was in a dose-dependent manner.

Baicalein had synergistic effects with gemcitabinel docetaxel on cell viability of pancreatic cancer cells. To determine whether baicalein has a synergistic effect with gemcitabine/docetaxel, we administered baicalein in combination with different concentrations of gemcitabine/ docetaxel to PANC-1. As shown in Fig. 2A, treatment with gemcitabine $(2 \mu \mathrm{M})$ mildly affected cell viability of PANC-1 with $82.03 \pm 5.033 \%$. When it was combined with baicalein $(10 \mu \mathrm{M})$, cell viability decreased to $36.5 \pm 2.848 \%$. Similarly, baicalein $(10 \mu \mathrm{M})$ plus gemcitabine $(5$ or $10 \mu \mathrm{M})$ remarkably affected cell viability compared with gemcitabine (5 and $10 \mu \mathrm{M})$ alone $(27.77 \pm 1.302 \%$ vs. $61.97 \pm 4.756 \%, \mathrm{P}<0.01$; $18.67 \pm 1.65 \%$ vs. $54.73 \pm 4.033 \%, \mathrm{P}<0.01)$. The $\mathrm{CI}$ values calculated by Calcusyn software were 0.191,0.204 and 0.179 when baicalein and gemcitabine were concurrently administered with the ratios of 5:1,2:1 and 1:1, respectively (Fig. 2C). Similar to gemcitabine, docetaxel also exhibited synergistic effect with baicalein. Treatment with baicalein $(10 \mu \mathrm{M})$ in combination with docetaxel $(2,5$, and $10 \mathrm{nM})$ resulted in remarkable decrease in cell viability when compared with docetaxel alone $(2,5$, and $10 \mathrm{nM})(39.17 \pm 2.109 \%$ vs. $64.2 \pm 2.969 \%$, $\mathrm{P}<0.01 ; 31.97 \pm 1.525 \%$ vs. $54.07 \pm 2.282 \%, \mathrm{P}<0.01$; $20.7 \pm 1.858 \%$ vs. $46.83 \pm 1.742 \%, \mathrm{P}<0.001$ ) (Fig. $2 \mathrm{~B}$ ). The CI values were $0.201,0.192$ and 0.099 , when baicalein $(\mu \mathrm{M})$ and docetaxel (nM) were administered with the ratios of 5:1, 2:1 and 1:1, respectively (Fig. 2D). Consistently, similar results were obtained from MIA $\mathrm{PaCa}-2$ and HPAF-II cells treatment with baicalein in combination with gemcitabine/ docetaxel (Fig. 2E-L), suggesting that baicalein has synergistic effects with gemcitabine/docetaxel on suppressing cell viability of PANC-1, MIA PaCa-2 and HPAF-II cells.

Combination treatment exhibited strong suppressive effects on the proliferation of PANC-1 cells. It is well-known that gemcitabine and docetaxel could inhibit proliferation of pancreatic cancer cells. However, whether baicalein has synergistic effects with gemcitabine/docetaxel on proliferation of pancreatic cancer cells is unknown. Therefore, we used EdU assay to determine the effects of cell proliferation by combination treatment. Relative proliferation ability was normalized to DMSO treated group by the ratios of proliferating cells which were stained red in the EdU assay. The relative proliferation ability of baicalein $(10 \mu \mathrm{M})$ treated group was lower than control, but no statistical significance (Fig. 3A and B). The relative proliferation ability of PANC-1 cells treated with baicalein $(10 \mu \mathrm{M})$ in combination with gemcitabine ( 2 and $10 \mu \mathrm{M})$ showed significantly decreased proliferation when compared to gemcitabine alone $(2$ and $10 \mu \mathrm{M})(2.677 \pm 2.677 \%$ vs. $17.48 \pm 3.243 \%, \mathrm{P}<0.05$; $1.496 \pm 0.8111 \%$ vs. $4.72 \pm 0.469 \%, \mathrm{P}<0.05$ ) (Fig. $3 \mathrm{~A}$ and $\mathrm{B}$ ). Similarly, the relative proliferation ability of cells treated with baicalein $(10 \mu \mathrm{M})$ in combination with docetaxel ( 2 and $10 \mathrm{nM})$ was significantly inhibited compared with docetaxel alone ( 2 and $10 \mathrm{nM})(29.36 \pm 5.822 \%$ vs. $45.11 \pm 8.995 \%$; 
A

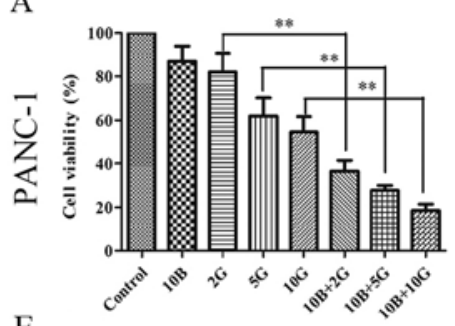

E
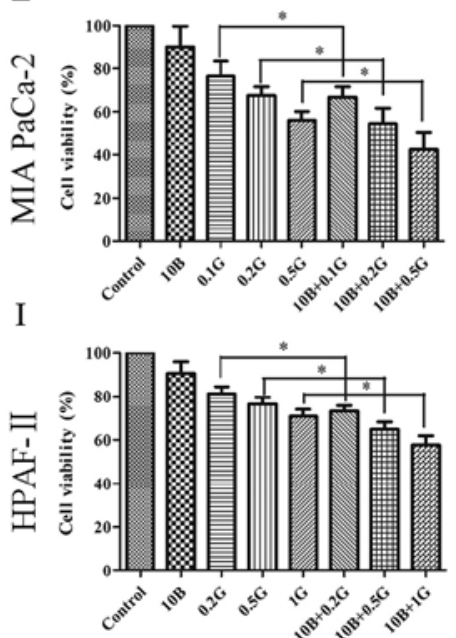

B
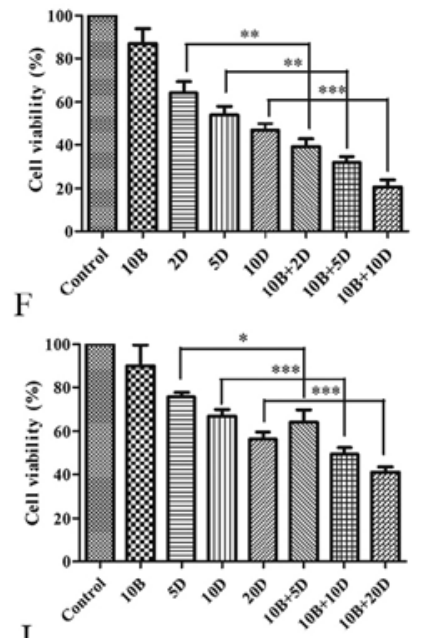

J

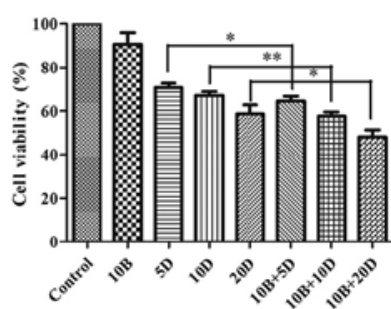

C

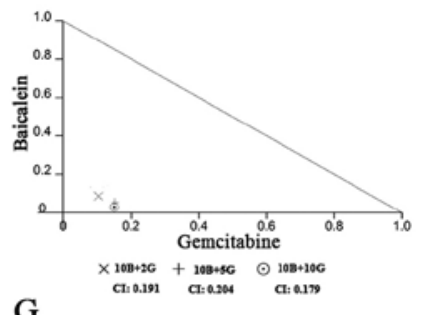

G
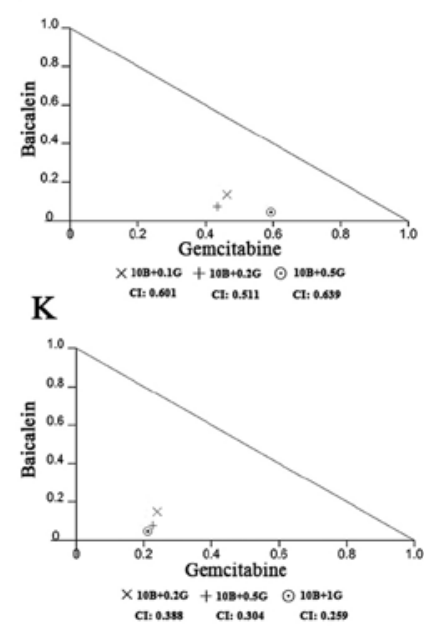

D

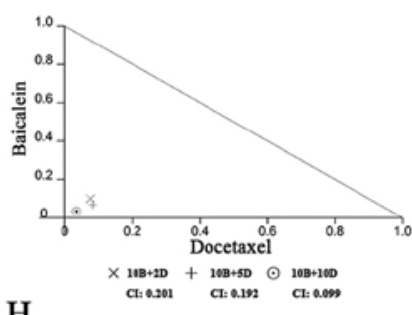

H
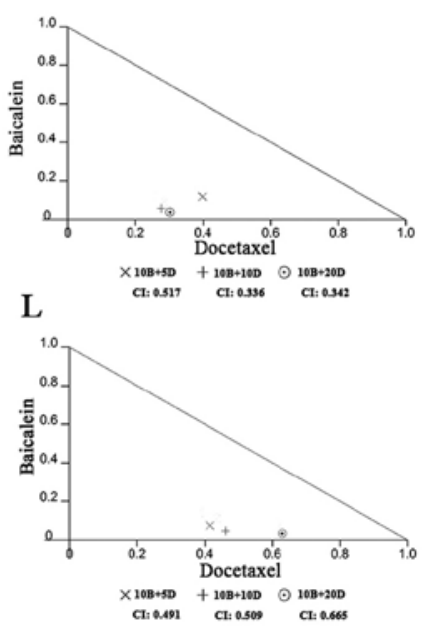

Figure 2. Effects of baicalein with gemcitabine or docetaxel on cell viability of PANC-1, MIA PaCa-2 and HPAF-II cells. (A and B) Combination effects of baicalein with gemcitabine/docetaxel on cell viability of PANC-1 cells were detected by MTT assay. Data are from three independent experiments. ${ }^{*}<0.05$, ${ }^{* *} \mathrm{P}<0.01$ and ${ }^{* * *} \mathrm{P}<0.001$ represent statistical significance. (C and D) Isobologram analysis assessing the synergism of baicalein with gemcitabine/docetaxel on PANC-1 cells; CI values depicting synergistic efficacy at indicated combination groups. (E and F) Combination effects of baicalein with gemcitabine/ docetaxel on cell viability of MIA PaCa-2 cells were detected by MTT assay. (G and H) Isobologram analysis assessing the synergism of baicalein with gemcitabine/docetaxel on MIA PaCa-2 cells; CI values describing synergistic effects at indicated combination groups. (I and J) Combinational effects of baicalein with gemcitabine/docetaxel on cell viability of HPAF-II cells were detected by MTT assay. (K and L) Isobologram analysis assessing the synergism of baicalein with gemcitabine/docetaxel on HPAF-II cells; CI values describing synergistic effects at indicated combination groups. $10 \mathrm{~B}, 10 \mu \mathrm{M}$ baicalein; $2 \mathrm{G}$, $2 \mu \mathrm{M}$ gemcitabine; $5 \mathrm{G}, 5 \mu \mathrm{M}$ gemcitabine; $10 \mathrm{G}, 10 \mu \mathrm{M}$ gemcitabine; $10 \mathrm{~B}+2 \mathrm{G}, 10 \mu \mathrm{M}$ baicalein plus $2 \mu \mathrm{M}$ gemcitabine; $10 \mathrm{~B}+5 \mathrm{G}, 10 \mu \mathrm{M}$ baicalein plus $5 \mu \mathrm{M}$ gemcitabine; $10 \mathrm{~B}+10 \mathrm{G}, 10 \mu \mathrm{M}$ baicalein plus $10 \mu \mathrm{M}$ gemcitabine; $2 \mathrm{D}, 2 \mathrm{nM}$ docetaxel; $5 \mathrm{D}, 5 \mathrm{nM}$ docetaxel; $10 \mathrm{D}, 10 \mathrm{nM}$ docetaxel; $10 \mathrm{~B}+2 \mathrm{D}, 10 \mu \mathrm{M}$ baicalein plus $2 \mathrm{nM}$ docetaxel; 10B+5D, $10 \mu \mathrm{M}$ baicalein plus $5 \mathrm{nM}$ docetaxel; 10B+10D, $10 \mu \mathrm{M}$ baicalein plus $10 \mathrm{nM}$ docetaxel.

$9.051 \pm 3.642 \%$ vs. $23.91 \pm 3.953 \%$ ), but no statistical significance (Fig. 3A and B). Our data suggest that baicalein might be a potential adjuvant to strengthen the anti-proliferation effects of the first-line clinical drugs on pancreatic cancer cells.

Combination treatment triggered cell cycle arrest of PANC-1 cells. Cell cycle blockade usually leads to growth inhibition of cancer cells. To investigate the effects of baicalein alone and combination treatment on cell cycle of PANC-1 cells, we conducted flow cytometry to analyze the proportion of different phases in cell cycle. The results showed that baicalein alone (50 and $100 \mu \mathrm{M}$ ) induced remarkable cell cycle arrest in the S-phase (42.95 and 45.74\%) compared with control (34.22\%) (Fig. 4A and C). Combination treatment with baicalein $(10 \mu \mathrm{M})$ and gemcitabine $(2 \mu \mathrm{M})$ resulted in a significant obvious change than gemcitabine $(2 \mu \mathrm{M})$ alone $(21.97$ vs. $6.46 \%)$. Similar results were observed between combination treatment with docetaxel $(2 \mathrm{nM})$ plus baicalein $(10 \mu \mathrm{M})$ and docetaxel ( $2 \mathrm{nM}$ ) alone (Fig. 4B and D). Therefore, baicalein might curb cell growth of PANC-1 by blocking the cell cycle.

Baicalein strengthened the inhibitory ability of gemcitabinel docetaxel on migration of PANC-1 cells. To further determine the effects of baicalein on cell migration, we used wound healing assay to assess the migration ability of PANC-1 cells. As expected, inhibitory effects of baicalein on migration of PANC-1 cells was in a dose-dependent manner. As shown in Fig. 5A and B, either 10 or $20 \mu \mathrm{M}$ baicalein alone exhibited no significant effects. However, baicalein at $50 \mu \mathrm{M}$ induced statistically significant reduction in recovery ratio of PANC-1 cells compared with control $(20 \pm 3.225 \%$ vs. $45.6 \pm 0.665 \%$; $P<0.01)$. Next, we investigated whether baicalein could reinforce the inhibitory ability of gemcitabine/docetaxel on migration of PANC-1 cells in vitro by using scratching assay. Treatment with baicalein $(10 \mu \mathrm{M})$ in combination with gemcitabine $(2 \mu \mathrm{M})$ showed more stronger ability of inhibiting cell migration compared with gemcitabine alone $(2 \mu \mathrm{M})(6.45 \pm 1.91 \%$ vs. $27.85 \pm 3.90 \%$; $\mathrm{P}<0.01)$. Similarly, combination of baicalein $(10 \mu \mathrm{M})$ and gemcitabine $(10 \mu \mathrm{M})$ exhibited significantly stronger suppressive effects on PANC-1 cells when compared with gemcitabine alone $(10 \mu \mathrm{M})(4.30 \pm 1.73 \%$ vs. $25.85 \pm 3.72 \%$; $\mathrm{P}<0.05)$. Baicalein $(10 \mu \mathrm{M})$ plus docetaxel $(10 \mathrm{nM})$ exhibited more obvious inhibitory effects than docetaxel alone $(10 \mathrm{nM})$ on migration of PANC-1 cells $(0.33 \pm 0.57 \%$ vs. $12.67 \pm 0.96 \%$; $\mathrm{P}<0.01)$. These results indicate that baicalein could enhance the capacity of gemcitabine/docetaxel to inhibit migration of PANC-1 cells. 

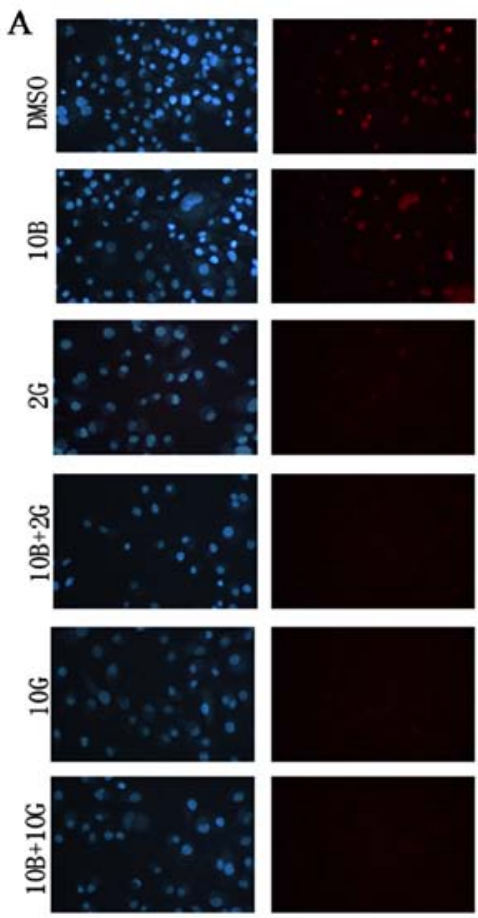
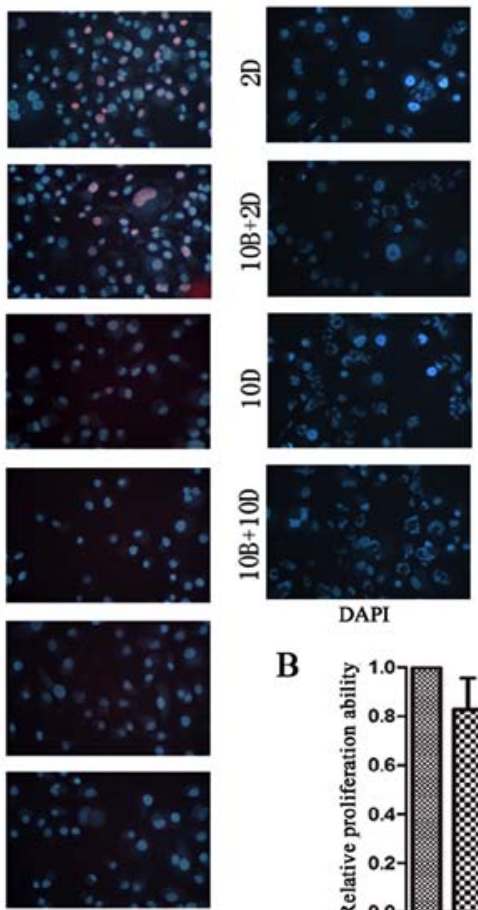

B
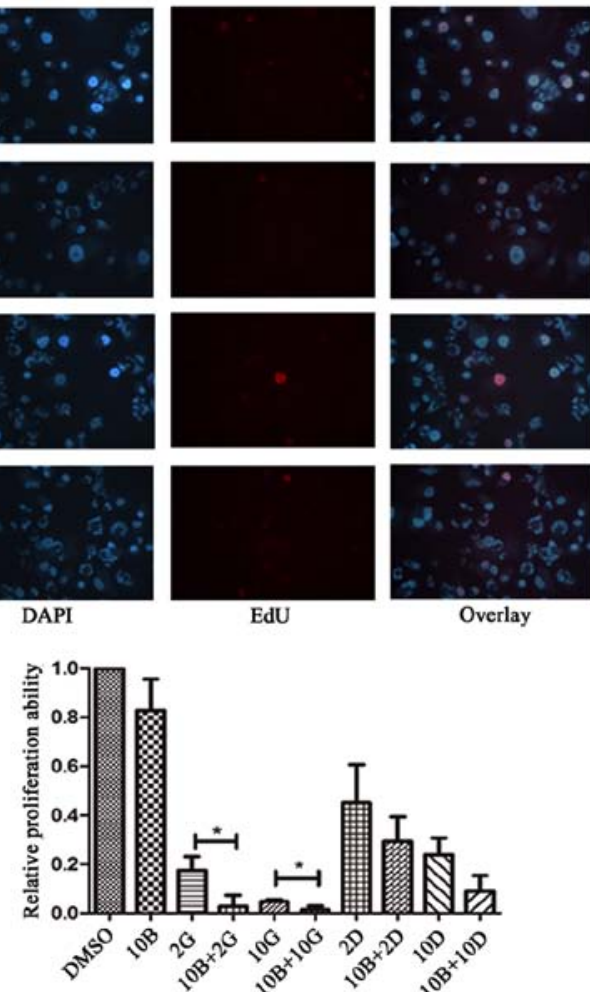

Figure 3. Effects of baicalein in combination with gemcitabine or docetaxel on proliferation of PANC-1 cells. (A) Proliferation of PANC-1 cells was detected by EdU assay. The nuclei of proliferating cells are stained red. (B) Percentage of nuclei in pink in the overlay image was used to evaluate relative proliferation ability of PANC-1 cells ( $\mathrm{P}<0.05)$.
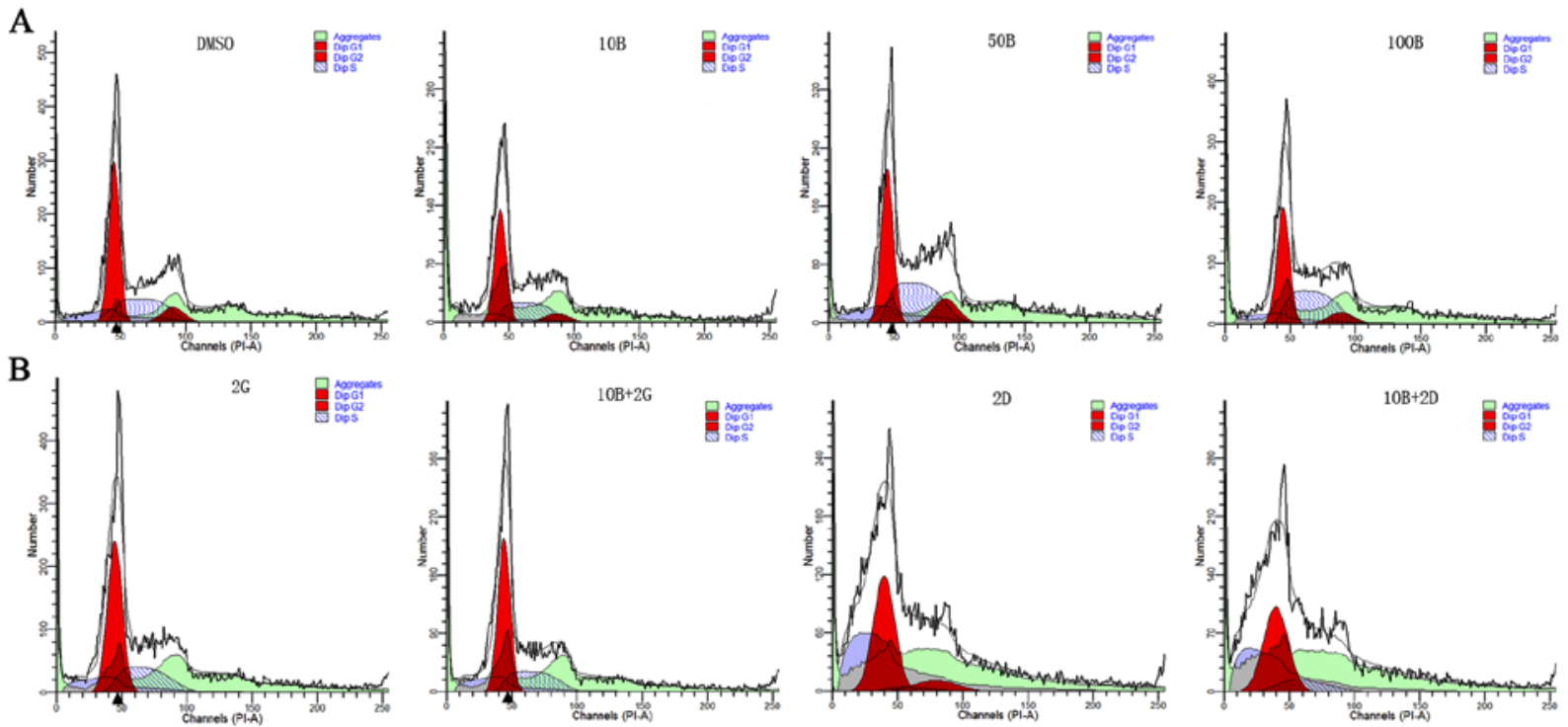

C

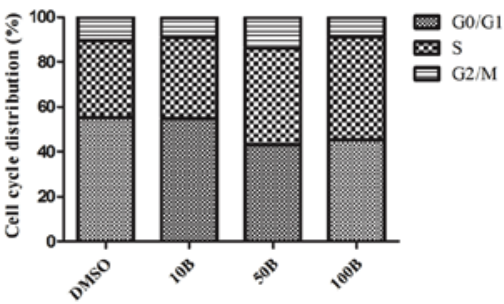

D

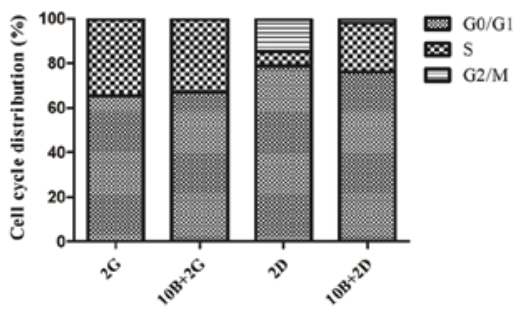

Figure 4. Baicalein in combination with gemcitabine or docetaxel affects the cell cycle of PANC-1 cells. (A) Cell cycle of PANC-1 cells treated with baicalein was analyzed by flow cytometry. (B) Cell cycle analysis of PANC-1 cells when PANC-1 cells were treated with gemcitabine or docetaxel alone or combined with baicalein at indicated concentrations. (C) Statistical analysis of different cell cycle phases when cells are treated with different concentrations of baicalein. (D) Quantitative statistical data of the cell cycle from (B). 50B, $50 \mu \mathrm{M}$ baicalein; 100B, $100 \mu \mathrm{M}$ baicalein. 
A

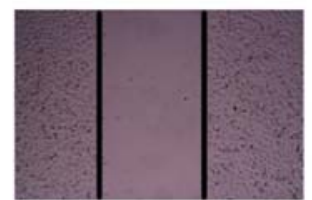

Control (0 h)

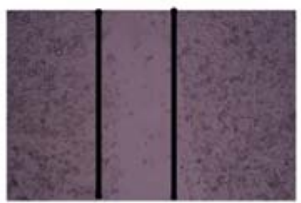

$10 B(24$ h)

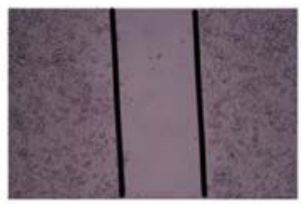

2D (24 h)

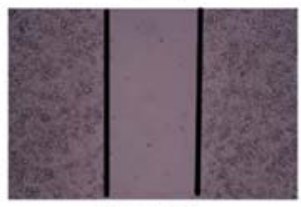

10D (24 h)

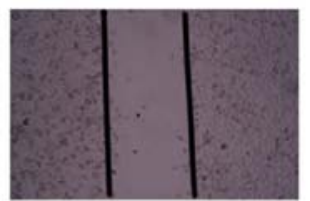

$20 B(24 \mathrm{~h})$

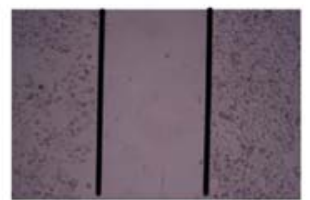

$10 \mathrm{~B}+2 \mathrm{D}(24 \mathrm{~h})$

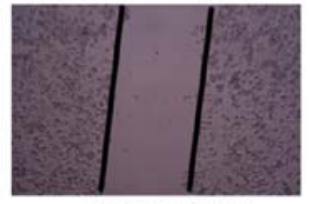

10B+10D (24 h)

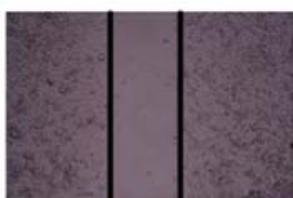

Control (24 h)

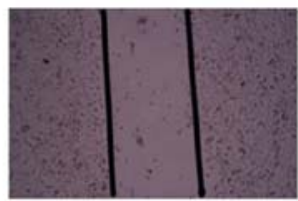

$50 \mathrm{~B}(24 \mathrm{~h})$

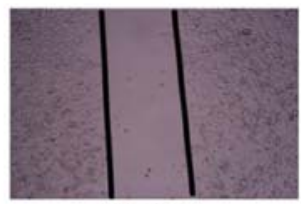

2G (24 h)

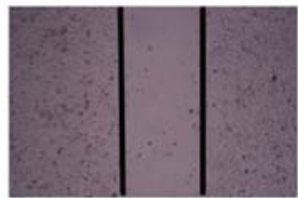

$10 \mathrm{G}(\mathbf{2 4} \mathrm{h})$

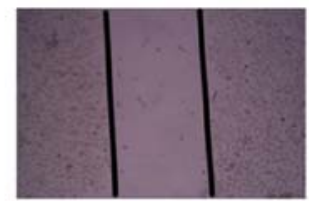

$100 \mathrm{~B}(24 \mathrm{~h})$

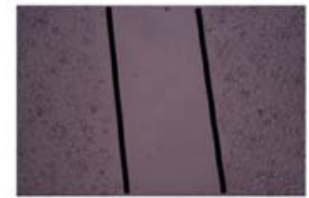

$10 \mathrm{~B}+2 \mathrm{G}(24 \mathrm{~h})$

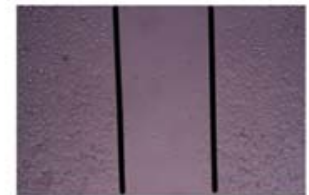

$10 \mathrm{~B}+10 \mathrm{G}(24 \mathrm{~h})$

B
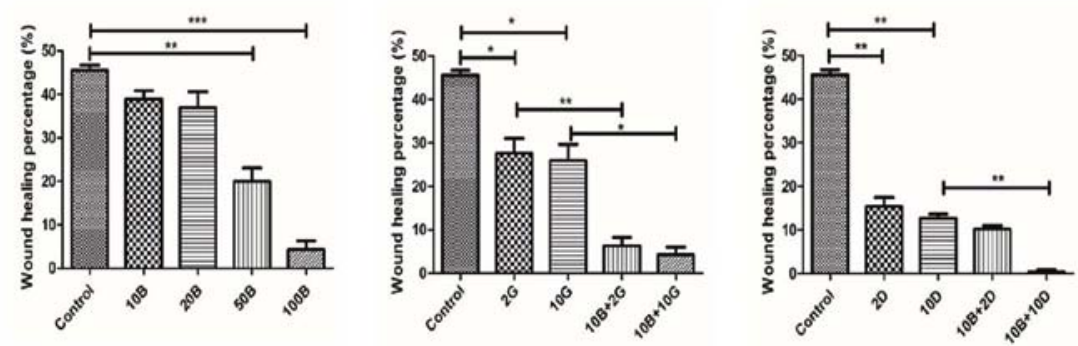

Figure 5. Effects of baicalein with gemcitabine or docetaxel on migration of PANC-1 cells. (A) Images of 'wounded' areas were obtained by inverted microscope at time points 0 and $24 \mathrm{~h}$. (B) Statistical analysis of wound healing percentage when PANC-1 cells were treated with indicated drug combinations. ${ }^{*} \mathrm{P}<0.05,{ }^{* *} \mathrm{P}<0.01$ and ${ }^{* * *} \mathrm{P}<0.001$ indicate statistically significant difference. $20 \mathrm{~B}, 20 \mu \mathrm{M}$ baicalein.

Baicalein induces apoptosis of PANC-1 cells and enhances the pro-apoptotic effects of docetaxel on PANC-1 cells. We further investigated whether baicalein-induced suppression of cell growth of PANC-1 cells is associated with cell apoptosis, we performed DAPI staining after treatment with drugs for $48 \mathrm{~h}$. As expected, treatment with baicalein at $10 \mu \mathrm{M}$ induced increased apoptosis in PANC-1 cells, compared with control $(1.922 \pm 0.551 \%$ vs. $0.377 \pm 0.132 \%, \mathrm{P}<0.05$; Fig. $6 \mathrm{~B})$. Then we further performed TEM to confirm the above results. Consistently, apoptotic change in PANC-1 cells was found after treatment with baicalein alone at $50 \mu \mathrm{M}$ (Fig. 6A). These data suggest that baicalein treatment could induce apoptosis of PANC-1 cells in vitro. To further uncover the molecular mechanism of apoptosis induced by baicalein, we performed western blot to detect the expression level of caspase-3, cleavedcaspase-3, PARP and cleaved-PARP, which are considered as classical apoptosis-related molecules. The results showed that, after treatment with baicalein, the expression level of caspase-3 was significantly decreased, accompanied by increased expression of cleaved-caspase-3. Immunoblotting for total PARP protein showed no significant change after baicalein treatment, while protein level of cleaved-PARP was markedly increased (Fig. 6E). Our data suggest that apoptosis-related caspase-3/PARP signaling pathway might play an important role in baicalein-induced apoptosis of PANC-1 cells.

Next, we further investigated whether baicalein could enhance pro-apoptotic effects of gemcitabine/docetaxel on PANC-1 cells using DAPI staining. Interestingly, treatment with $10 \mu \mathrm{M}$ baicalein in combination with $2 \mathrm{nM}$ docetaxel resulted in significantly increased apoptosis of PNCA-1 cells compared with $2 \mathrm{nM}$ docetaxel alone $(75.86 \pm 5.184 \%$ vs. $15.13 \pm 2.169 \% ; \mathrm{P}<0.001)$. Similar results were observed at high concentrations of docetaxel. Docetaxel at $10 \mathrm{nM}$ triggered significant apoptosis of PNCA-1 cells with the apoptosis rate of $76.07 \pm 9.61 \%$, while $10 \mathrm{nM}$ docetaxel together with $10 \mu \mathrm{M}$ baicalein induced more severe cell apoptosis of $87.63 \pm 5.19 \%(\mathrm{P}<0.05)$ (Fig. 6B and $\mathrm{D})$. In addition, TEM showed that apoptosis changes existed in all drug treated groups (Fig. 6A). These results demonstrate that baicalein has a potential to enhance pro-apoptotic effects of docetaxel on PANC-1 cells in vitro. However, treatment with $10 \mu \mathrm{M}$ baicalein plus $2 \mu \mathrm{M}$ gemcitabine did not induce significant change in apoptosis rate of PANC-1 cells when compared with $2 \mu \mathrm{M}$ gemcitabine alone. Similarly, there was also no significant 
A
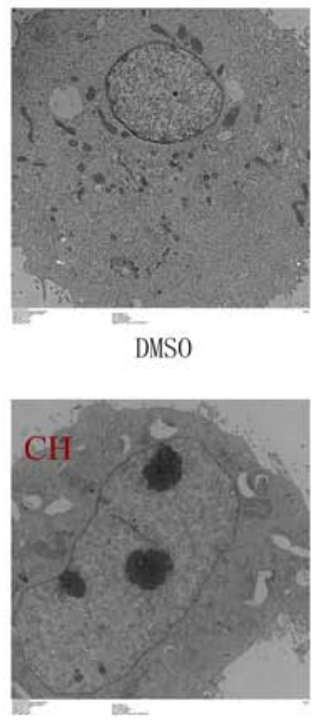

$50 \mathrm{~B}$

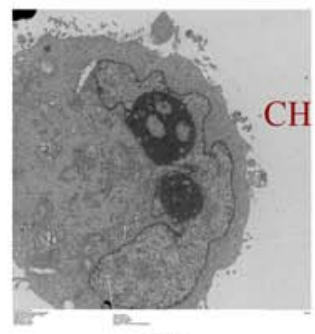

$10 \mathrm{G}$

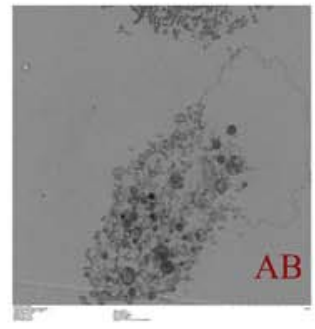

$10 \mathrm{~B}+10 \mathrm{G}$

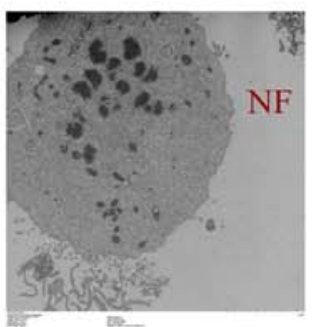

10D

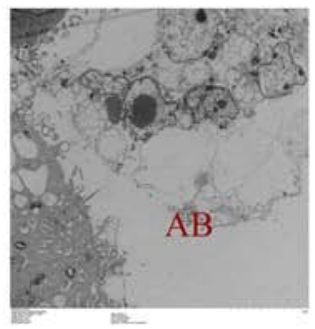

$10 \mathrm{~B}+10 \mathrm{D}$
B

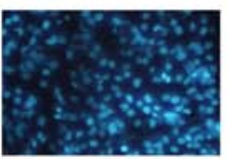

DMSO

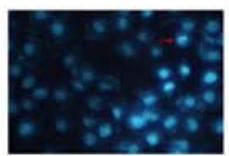

2G

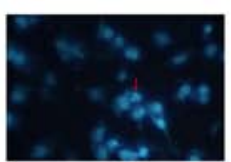

10G

C
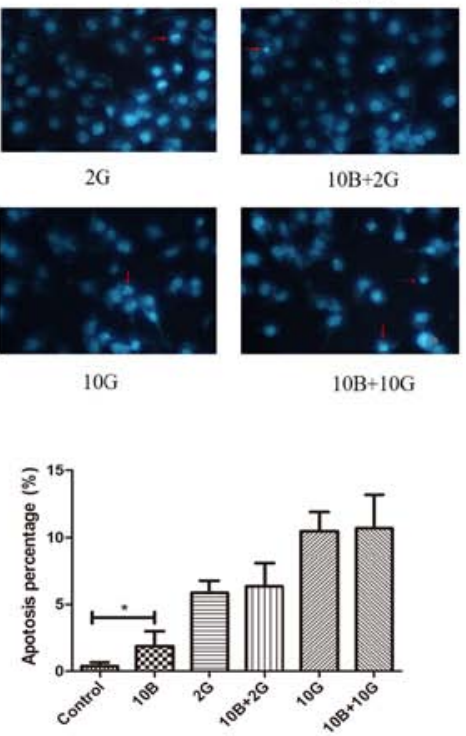

$10 B+2 G$

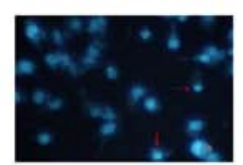

$10 \mathrm{~B}+10 \mathrm{G}$

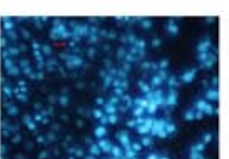

$10 \mathrm{~B}$

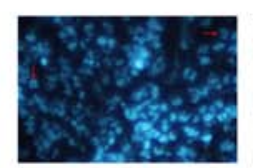

2D

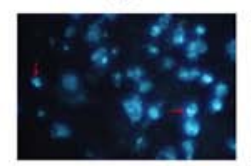

$10 \mathrm{D}$

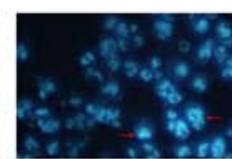

$10 \mathrm{~B}+2 \mathrm{D}$

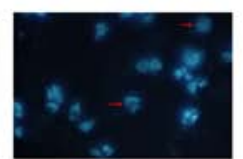

$10 \mathrm{~B}+10 \mathrm{D}$

D

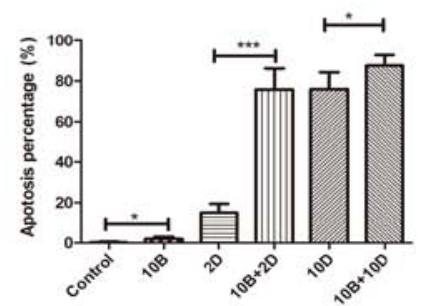

E
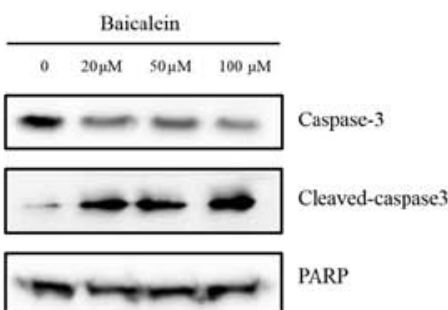

$\longrightarrow \sim-\infty$ Cleaved-PARP

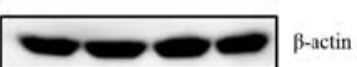

F

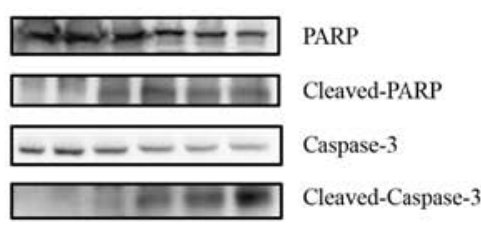

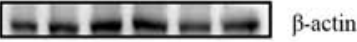

DMSO $\quad 10 \mathrm{~B} \quad 2 \mathrm{G} \quad 10 \mathrm{~B}+2 \mathrm{G} \quad 10 \mathrm{G} \quad 10 \mathrm{~B}+10 \mathrm{G}$

G

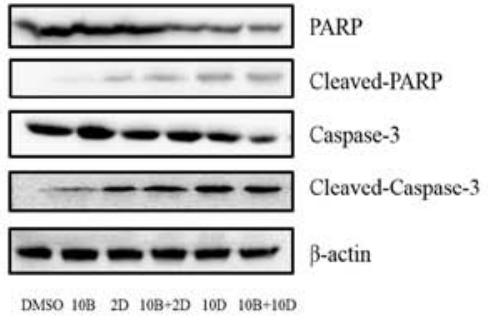

Figure 6. Effects of baicalein with gemcitabine or docetaxel on apoptosis of PANC-1 cells. (A) Apoptotic changes were observed using TEM in the indicated groups. AB, apoptotic bodies; $\mathrm{CH}$, chromatin condensation; NF, nucleus fragmentation. (B) Apoptotic morphological change including nuclear condensation and fragmentation observed by DAPI staining experiment. The red arrow represents typical nuclear changes of apoptotic cells. (C and D) Statistical analysis of percentage in cell apoptosis in different treated groups ( ${ }^{*} \mathrm{P}<0.05$ and $\left.{ }^{* * *} \mathrm{P}<0.001\right)$. (E) Expression of caspase-3, cleaved-caspase-3, PARP and cleaved-PARP detected by western blotting. $\beta$-actin was used as internal control. (F) Immunoblot analysis of caspase-3, cleaved-caspase-3, PARP and cleaved-PARP of PANC-1 cells after treatment with solvent (DMSO), baicalein $(10 \mu \mathrm{M})$ alone, gemcitabine $(2$ or $10 \mu \mathrm{M})$ alone and combination treatment $(10 \mu \mathrm{M}$ baicalein + $2 \mu \mathrm{M}$ gemcitabine or $10 \mu \mathrm{M}$ baicalein $+10 \mu \mathrm{M}$ gemcitabine). (G) Immunoblot analysis of caspase-3, cleaved-caspase-3, PARP and cleaved-PARP of PANC-1 cells after treatment with solvent (DMSO), baicalein $(10 \mu \mathrm{M})$ alone, docetaxel $(2$ or $10 \mathrm{nM})$ alone and combination treatment $(10 \mu \mathrm{M}$ baicalein $+2 \mathrm{nM}$ docetaxel or $10 \mu \mathrm{M}$ baicalein $+10 \mathrm{nM}$ docetaxel).

difference in apoptosis of PNCA-1 cells treated with $10 \mu \mathrm{M}$ baicalein plus $10 \mu \mathrm{M}$ gemcitabine compared with $10 \mu \mathrm{M}$ gemcitabine alone (Fig. 6B and C). Moreover, caspase-3/PARP signaling pathway was significantly activated when treated with baicalein in combination with gemcitabine/docetaxel (Fig. 6F and G). These data suggest that baicalein might exert different effects on pro-apoptosis effect of different drugs and the underlying mechanism might be complicated.

\section{Discussion}

As an enigmatic and aggressive malignancy with a dismal prognosis, PC is the fourth leading cause of cancer-related death in the United States. The current first-line chemotherapy regimens, including gemcitabine and paclitaxel remain unsatisfactory to OS of PC patients $(18,19)$. Gemcitabine and docetaxel have been widely used in PC, but the median OS 
under gemcitabine therapy was only 5.65 months (18-20). Therefore, an alternative approach to the treatment for PC is urgently needed. Baicalein, a kind of low toxic natural compound, has been reported to exert antitumor effects on many types of cancer including PC. However, effects of combination therapy of baicalein with gemcitabine/docetaxel on PC is unclear. In the current study, we are the first to report that baicalein has synergistic effects with gemcitabine/docetaxel on PANC-1, MIA PaCa-2 and HPAF-II cells, suggesting that baicalein might be an alternative choice in combination treatment for PC.

Although $10 \mu \mathrm{M}$ baicalein alone exhibited mild impact on the proliferation of PC cells, combination treatment of baicalein with gemcitabine/docetaxel resulted in obvious suppression of cell proliferation. Cell cycle arrest is one of the most important cellular mechanisms leading to proliferation inhibition of tumor cells. Our study showed that high dose of baicalein alone (50 or $100 \mu \mathrm{M}$ ) could result in cell cycle arrest of PC cells in $\mathrm{S}$ phase. This is in accordance with previous studies in which baicalein alone could lead to G0/G1, G2/M and S-phase arrest in various cancer cells (14,21-24). In addition, combination treatment of baicalein with docetaxel increased approximately $15 \%$ ratio of S-phase of PC cells. The cell cycle change caused by baicalein gave further explanations to its capacity to inhibit cell growth of PC cells.

Metastasis is another critical factor associated with the poor OS in PC patients. Therefore, investigators have tried new combination drug therapy regimens in order to prolong OS of metastatic PC patients $(5,6,25,26)$. We conducted wound healing assay to evaluate migration ability of PC cells in the presence of baicalein at different concentrations or in combination treatment. The results not only revealed the potential of baicalein at high concentrations to inhibit migration of PC cells, but also indicated that it might be a potent adjuvant benefiting the inhibitory ability of gemcitabine/docetaxel on migration of PC cells. Therefore, baicalein might be a promising drug to improve the outcome of current chemotherapy regimen for metastatic $\mathrm{PC}$.

It has been reported that baicalein can induce apoptosis in many types of cancer $(19,27-29)$. Consistently, in the current study, PC cells treated with baicalein suffered from obvious apoptotic change. This effect might be dependent on caspase-3/PARP signaling pathway. Furthermore, baicalein was found to strengthen the pro-apoptotic effects of docetaxel on PC cells. Curiously, the synergistic effects of baicalein with gemcitabine on PC cells seem to be independent on caspase-3/ PARP signaling pathway. Therefore, its underlying mechanism needs to be further investigated.

In conclusion, our study demonstrate that baicalein has synergistic effects with gemcitabine/docetaxel on the proliferation, cell cycle, migration and apoptosis of PC cells. These data suggest that combination treatment containing baicalein might be a promising strategy to improve the outcome of clinical treatment for PC.

\section{Acknowledgements}

We would like to thank Dr Panwen Wang (Mayo Clinics, Rochester, MN, USA) for the revision of the manuscript. This study was supported by National Natural Foundation of China (no. 81500151), Wuhan Program (no. WX15Z03 and A201113) and Undergraduate Training Program for Innovation and Entrepreneurship by Wuhan University (no. S2016861).

\section{References}

1. Siegel RL, Miller KD and Jemal A: Cancer statistics, 2016. CA Cancer J Clin 66: 7-30, 2016.

2. Hidalgo M: Pancreatic cancer. N Engl J Med 362: 1605-1617, 2010.

3. Humphris JL, Johns AL, Simpson SH, Cowley MJ, Pajic M, Chang DK, Nagrial AM, Chin VT, Chantrill LA, Pinese M, et al; Australian Pancreatic Cancer Genome Initiative: Clinical and pathologic features of familial pancreatic cancer. Cancer 120: 3669-3675, 2014

4. Hidalgo M: New insights into pancreatic cancer biology. Ann Oncol 23 (Suppl 10): x135-x138, 2012.

5. Garrido-Laguna I and Hidalgo M: Pancreatic cancer: From stateof-the-art treatments to promising novel therapies. Nat Rev Clin Oncol 12: 319-334, 2015.

6. Von Hoff DD, Ervin T, Arena FP, Chiorean EG, Infante J, Moore M, Seay T, Tjulandin SA, Ma WW, Saleh MN, et al: Increased survival in pancreatic cancer with nab-paclitaxel plus gemcitabine. N Engl J Med 369: 1691-1703, 2013.

7. Yassine F, Salibi E and Gali-Muhtasib H: Overview of the formulations and analogs in the taxanes' story. Curr Med Chem 23: 4540-4558, 2016.

8. Mantripragada KC and Safran H: Optimizing initial chemotherapy for metastatic pancreatic cancer. Future Oncol 12: 1125-1133, 2016.

9. Liu H, Dong Y, Gao Y, Du Z, Wang Y, Cheng P, Chen A and Huang H: The fascinating effects of baicalein on cancer: A review. Int J Mol Sci 17: 17, 2016.

10. Donald G, Hertzer K and Eibl G: Baicalein - an intriguing therapeutic phytochemical in pancreatic cancer. Curr Drug Targets 13: 1772-1776, 2012.

11. Wu JY, Tsai KW, Li YZ, Chang YS, Lai YC, Laio YH, Wu JD and Liu YW: Anti-bladder-tumor effect of baicalein from Scutellaria baicalensis Georgi and its application in vivo. Evid Based Complement Alternat Med 2013: 579751, 2013.

12. Taniguchi H, Yoshida T, Horinaka M, Yasuda T, Goda AE, Konishi M, Wakada M, Kataoka K, Yoshikawa T and Sakai T: Baicalein overcomes tumor necrosis factor-related apoptosisinducing ligand resistance via two different cell-specific pathways in cancer cells but not in normal cells. Cancer Res 68: 8918-8927, 2008.

13. Wang L, Ling Y, Chen Y, Li CL, Feng F, You QD, Lu N and Guo QL: Flavonoid baicalein suppresses adhesion, migration and invasion of MDA-MB-231 human breast cancer cells. Cancer Lett 297: 42-48, 2010.

14. Wang CZ, Zhang CF, Chen L, Anderson S, Lu F and Yuan CS: Colon cancer chemopreventive effects of baicalein, an active enteric microbiome metabolite from baicalin. Int J Oncol 47: 1749-1758, 2015.

15. Li-Weber M: New therapeutic aspects of flavones: The anticancer properties of Scutellaria and its main active constituents Wogonin, Baicalein and Baicalin. Cancer Treat Rev 35: 57-68, 2009.

16. Lu QY, Zhang L, Moro A, Chen MC, Harris DM, Eibl G and Go VL: Detection of baicalin metabolites baicalein and oroxylina in mouse pancreas and pancreatic xenografts. Pancreas 41: 571-576, 2012.

17. Takahashi H, Chen MC, Pham H, Angst E, King JC, Park J, Brovman EY, Ishiguro H, Harris DM, Reber HA, et al: Baicalein, a component of Scutellaria baicalensis, induces apoptosis by Mcl-1 down-regulation in human pancreatic cancer cells. Biochim Biophys Acta 1813: 1465-1474, 2011

18. Caparello C, Meijer LL, Garajova I, Falcone A, Le Large TY, Funel N, Kazemier G, Peters GJ, Vasile E and Giovannetti E: FOLFIRINOX and translational studies: Towards personalized therapy in pancreatic cancer. World J Gastroenterol 22: 6987-7005, 2016.

19. Goel G and Sun W: Novel approaches in the management of pancreatic ductal adenocarcinoma: Potential promises for the future. J Hematol Oncol 8: 44, 2015.

20. Ghosn M, Ibrahim T, Assi T, El Rassy E, Kourie HR and Kattan J: Dilemma of first line regimens in metastatic pancreatic adenocarcinoma. World J Gastroenterol 22: 10124-10130, 2016. 
21. Mu J, Liu T, Jiang L, Wu X, Cao Y, Li M, Dong Q, Liu Y and $\mathrm{Xu} \mathrm{H}$ : The traditional Chinese medicine baicalein potently inhibits gastric cancer cells. J Cancer 7: 453-461, 2016.

22. Zheng YH, Yin LH, Grahn TH, Ye AF, Zhao YR and Zhang QY: Anticancer effects of baicalein on hepatocellular carcinoma cells. Phytother Res 28: 1342-1348, 2014.

23. Seo MJ, Choi HS, Jeon HJ, Woo MS and Lee BY: Baicalein inhibits lipid accumulation by regulating early adipogenesis and m-TOR signaling. Food Chem Toxicol 67: 57-64, 2014.

24. Wang CZ, Calway TD, Wen XD, Smith J, Yu C, Wang Y, Mehendale SR and Yuan CS: Hydrophobic flavonoids from Scutellaria baicalensis induce colorectal cancer cell apoptosis through a mitochondrial-mediated pathway. Int J Oncol 42: 1018-1026, 2013

25. Kosmidis C, Sapalidis K, Kotidis E, Mixalopoulos N Zarogoulidis P, Tsavlis D, Baka S, Man YG and Kanellos J: Pancreatic cancer from bench to bedside: Molecular pathways and treatment options. Ann Transl Med 4: 165, 2016.
26. Lemstrova R, Melichar B and Mohelnikova-Duchonova B: Therapeutic potential of taxanes in the treatment of metastatic pancreatic cancer. Cancer Chemother Pharmacol 78: 1101-1111, 2016.

27. Gong WY, Zhao ZX, Liu BJ, Lu LW and Dong JC: Exploring the chemopreventive properties and perspectives of baicalin and its aglycone baicalein in solid tumors. Eur J Med Chem 126: 844-852, 2017.

28. Li J, Ma J, Wang KS, Mi C, Wang Z, Piao LX, Xu GH, Li X, Lee JJ and Jin X: Baicalein inhibits TNF- $\alpha$-induced NF- $\kappa B$ activation and expression of NF- $\mathrm{BB}$-regulated target gene products Oncol Rep 36: 2771-2776, 2016.

29. Choi EO, Park C, Hwang HJ, Hong SH, Kim GY, Cho EJ, Kim WJ and Choi YH: Baicalein induces apoptosis via ROS-dependent activation of caspases in human bladder cancer 5637 cells. Int J Oncol 49: 1009-1018, 2016. 